\title{
Astragalus Polysaccharide Regulates Proliferation, Migration and Invasion of Lung Cancer Cells via Janus Kinase/Signal Transducer and Activator of Transcription Signaling Pathway
}

\author{
H. CAI AND J. CAO*
}

Department of Respiratory Medicine, Jiangnan Hospital Affiliated to Zhejiang University of Traditional Chinese Medicine, Hangzhou, Zhejiang Province 311200, China

Cai et al.: Astragalus Polysaccharide and Lung Cancer

\begin{abstract}
To evaluate the regulatory effects of Astragalus polysaccharide on the proliferation, migration and invasion of lung cancer cells via the Janus kinase/signal transducer and activator of transcription signaling pathway. The inhibitory effects of treatment with Astragalus polysaccharide at different concentrations $(0,0.5,1.0,2.0$, 4.0, 8.0 and $16.0 \mathrm{mg} / \mathrm{ml})$ for different time $(24,48$ and $72 \mathrm{~h})$ on the proliferation of adenocarcinomic human alveolar basal epithelial cells in the logarithmic growth phase were detected by methyl thiazolyl tetrazolium assay and its inhibitory effects on the migration and invasion of adenocarcinomic human alveolar basal epithelial cells were determined by wound healing assay and Transwell migration and invasion assays respectively. In contrast with control group, the proliferation of adenocarcinomic human alveolar basal epithelial cells treated with different concentrations of Astragalus polysaccharide for 24, 48 and $72 \mathrm{~h}$ was remarkably inhibited in a dose and time dependent manner under the same drug concentration, longer the action time, higher the proliferation inhibition rate of adenocarcinomic human alveolar basal epithelial cells; under the same action time, higher the drug concentration, higher the proliferation inhibition rate of adenocarcinomic human alveolar basal epithelial cells. The number of migrating cells dropped significantly in Astragalus polysaccharide groups $(\mathbf{p}<\mathbf{0 . 0 5})$, which was more obvious with the increase of Astragalus polysaccharide concentration $(\mathbf{p}<0.05)$. Astragalus polysaccharide can dramatically inhibit the proliferation of lung cancer adenocarcinomic human alveolar basal epithelial cells in a concentration and time-dependent manner and also effectively suppress their migration and invasion. The mechanism may be related to its ability to up-regulate the protein expressions of Src homology region 2 containing protein tyrosine phosphatase 2 and suppressor of cytokine signaling 3 and inhibit the over-activation of the Janus kinase/signal transducer and activator of transcription signaling pathway in lung cancer adenocarcinomic human alveolar basal epithelial cells.
\end{abstract}

Key words: Astragalus polysaccharide, lung cancer, Janus kinase, chemotherapy, surgery

According to the World Cancer Report issued by the International Agency for Research on Cancer (IARC, a part of the World Health Organization), cancer has been a major threat to public health in both developing and developed countries. About 7.60 million people died of cancer in $2008^{[1]}$ and it is estimated that more than 11 million people will die of cancer in 2030 based on the current trend. Lung cancer is the most common primary lung malignant tumor with an increasing incidence and high mortality and the existing therapies of lung cancer include surgery, chemotherapy, radiotherapy, Traditional Chinese Medicine (TCM) therapy and

*Address for correspondence

E-mail: cao.jianbiao@163.com

November-December 2021 molecular targeted therapy ${ }^{[2]}$. The treatment of early lung cancer is dominated by surgery. However, about $80 \%$ of patients have been in middle advanced stage upon diagnosis. At this time, distant metastasis has occurred to cancer cells and chemotherapy is the best therapy, but chemotherapy will inhibit the bone

\footnotetext{
This is an open access article distributed under the terms of the Creative Commons Attribution-NonCommercial-ShareAlike 3.0 License, which allows others to remix, tweak, and build upon the work non-commercially, as long as the author is credited and the new creations are licensed under the identical terms
}

Accepted 08 November 2021

Revised 15 June 2021

Received 08 May 2020

Indian J Pharm Sci 2021;83(6):1215-1220 
marrow hematopoietic system and cause leucopenia and thrombocytopenia, making the body prone to accompanying infections and even death ${ }^{[3]}$. Meanwhile, radiotherapy is accompanied by many complications as well, showing bad effects on patients with advanced lung cancer and is not applicable to patients with poor constitution and older age. As a result, some Chinese herbal medicines derived from natural plants have become a research hotspot of lung cancer therapy in recent years, which present anti-tumor effects and no or little toxicity ${ }^{[4,5]}$. Astragalus Poly Saccharide (APS) is a polysaccharide component extracted from the Astragalus root and it is the main water-soluble active component. According to recent studies, APS has important anti-tumor effects ${ }^{[6]}$. This study aimed to investigate the effects of APS on the proliferation and apoptosis of adenocarcinomic human alveolar basal epithelial cells (A549 cells), so as to provide new ideas for the therapy of lung cancer.

\section{MATERIALS AND METHODS}

\section{Materials:}

In this study, the A549 human lung cancer cell lines were purchased from the Cell Bank of Type Culture Collection Committee of Chinese Academy of Sciences and APS was sourced from Tianjin Cinorch Pharmaceutical Co., Ltd. High-glucose Dulbecco's Modified Eagle Medium (DMEM) was bought from HyClone (USA) and Fetal Bovine Serum (FBS) was sourced from Zhejiang Tianhang Biotechnology Co., Ltd. Methyl Thiazolyl Tetrazolium (MTT) and the Radio Immuno Precipitation Assay (RIPA) lysate were purchased from Sigma (USA), penicillin and streptomycin were provided by Beyotime Biotechnology. Bicinchoninic Acid (BCA) protein assay kits were purchased from Applygen Technologies Inc., and prestained protein markers and Trizol were sourced from Thermo Fisher Scientific (USA). Polyvinylidene Fluoride (PVDF) membranes were purchased from Millipore (USA). The monoclonal antibodies of rabbit anti-human Suppressors of Cytokine Signaling 3 (SOCS3), rabbit anti-human Src Homology-2 Domain-Containing Protein Tyrosine Phosphatase-2 (SHP2) and rabbit anti-human IMAS1 were bought from CST (USA). Mouse anti-human monoclonal antibodies, goat antirabbit Immunoglobulin G (IgG) and goat anti-mouse IgG labeled with Horseradish Peroxidase (HRP) were purchased from Beijing Zhongshan Golden Bridge Biotechnology Co., Ltd.

\section{Cell culture:}

Cells in the logarithmic phase were selected and cultured with high-glucose DMEM containing $10 \%$ FBS, $100 \mathrm{U} / \mathrm{ml}$ penicillin and $0.1 \mathrm{mg} / \mathrm{ml}$ streptomycin at $37^{\circ}$ and $5 \%$ Carbon Dioxide $\left(\mathrm{CO}_{2}\right)$. Besides, $0.25 \%$ trypsin was applied for cell passage.

\section{Cell proliferation detected by MTT assay:}

The stably passaged A549 cells were digested and the concentration of the cell suspension was adjusted to $1 \times 10^{4}$. The cells were inoculated into 96-well plates and $200 \mu \mathrm{l}$ of culture medium was added to each well of each plate. 6 replicates were set for each concentration and the APS at the concentration of $0,0.5,1.0,2.0,4.0$, 8.0 and $16.0 \mathrm{mg} / \mathrm{ml}$ were added to each well for reaction for 24,48 and $72 \mathrm{~h}$ respectively. Next, $200 \mu \mathrm{l}$ of MTT $(5 \mathrm{mg} / \mathrm{ml})$ was added to each well. After standing for $4 \mathrm{~h}$, the cells were taken out, collected and centrifuged for $10 \mathrm{~min}$ at $1000 \mathrm{r} / \mathrm{min}$. Then, the culture medium in the wells was sucked out carefully and $200 \mu \mathrm{l}$ of Dimethyl Sulfoxide (DMSO) was added to each well. After shaking for $10 \mathrm{~min}$, the absorbance of each well was detected at Optical Density (OD) $490 \mathrm{~nm}$ using an Enzyme Linked Immunosorbent Assay (ELISA) reader. Afterwards, the cell inhibition rate of each well was calculated as follows: Inhibition rate $=[1-($ mean OD490 value of wells in medication group-OD490 value of the zero adjustment well)/(mean OD490 value of wells in control group-OD490 value of the zero adjustment well) $] \times 100 \%$. The assay was repeated for 3 times and the mean value was taken.

\section{Wound healing assay:}

The stably growing A549 cells were conventionally digested, centrifuged, collected and counted and the cells were inoculated into a total of 6 wells on a 24well plate at $1 \times 10^{4} /$ well. When the cells reached $80 \%$ confluence, a wound was scratched on the cells using a plastic pipette tip. Later, 3 wells were randomly selected as the experimental group, which were added with DMEM complete medium containing $4.0 \mathrm{mg} /$ $\mathrm{ml}$ APS, while the other 3 wells were used as control group. In control group, the cells were cultured in the conventional medium. After conventional culture in an incubator for 0 and $48 \mathrm{~h}$ respectively, the wound healing status in experimental group and control group at different time points was observed and the healing rate was calculated as follows: healing rate $=$ (wound size at $0 \mathrm{~h}$-wound size at $48 \mathrm{~h}$ )/wound size at $0 \mathrm{~h}$ $\times 100 \%$. The assay was repeated for 3 times and the 
mean value was taken.

\section{Cell migration ability assay:}

A549 lung cancer cells were treated with APS at the concentration of $0,2.0$ and $4.0 \mathrm{mg} / \mathrm{ml}$ respectively and the cell migration ability at $48 \mathrm{~h}$ was detected in the Transwell chamber. Later, the cells in the middle and lower chambers of each group were collected and the number of migrating cells was counted under a microscope.

\section{Cell invasion ability assay:}

DMEM was used for dilution and preparation of Matrigel, and it was added to the 24-well Transwell chamber. A549 lung cancer cells were treated with APS at the concentration of $0,2.0$ and $4.0 \mathrm{mg} / \mathrm{ml}$ for $16 \mathrm{~h}$ respectively. Later, the cell invasion ability was detected in the Transwell chamber and the number of invasive cells was observed under a microscope.

\section{Detection of relative protein expressions by western blotting:}

A549 cells were treated with APS at the concentration of $0,2.0$ and $4.0 \mathrm{mg} / \mathrm{ml}$ for $24 \mathrm{~h}$ respectively and the protein expressions of SHP2, SOCS3 and Protein Inhibitor of Activated Stat 1 (PIAS1) were detected by Western blotting. After the collected cells were lysed and the protein concentration was determined, the loading buffer was added to the lysate, which was heated at $1000^{\circ}$ and boiled for $10 \mathrm{~min}$. Later, each well was loaded with $40 \mu \mathrm{g}$ of proteins for electrophoresis, which was subjected to electrophoretic separation in $12 \%$ Sodium Dodecyl Sulphate-Polyacrylamide Gel Electrophoresis (SDS-PAGE) gel. The voltage was set to $70 \mathrm{~V}$ during the electrophoretic separation of spacer gel and $120 \mathrm{~V}$ during the electrophoretic separation of separation gel. After separation, the proteins were transferred on to membranes at $275 \mathrm{~mA}$ for $60 \mathrm{~min}$ and then the Nitrocellulosee (NC) membranes were sealed with $5 \%$ skim milk powder at room temperature for $1 \mathrm{~h}$. After incubation with primary antibodies overnight and secondary antibodies at room temperature for $1 \mathrm{~h}$, the membranes were washed for 3 times, followed by color development and imaging.

\section{Statistical analysis:}

Statistical Package for the Social Sciences (SPSS) 26.0 software was utilized for statistical analysis, and the measurement data in line with normal distribution were expressed by mean \pm standard deviation. After variance analysis, Least significant difference test was performed for comparisons between groups, $\mathrm{p}<0.05$ indicated that a difference was statistically significant.

\section{RESULTS AND DISCUSSION}

Compared with control group, APS at different concentrations all showed significant inhibitory effects on the proliferation of A549 cells at 24, 48 and $72 \mathrm{~h}$ in a dose and time dependent manner. At the same drug concentration, the longer the action time, the higher the inhibition rate of A549 cell proliferation. With the same action time, the higher the drug concentration, the higher the inhibition rate of A549 cell proliferation (fig. 1).

The wound healing speed of A549 cells was lower in ASP groups than that in control group. The cells in control group almost fully healed after $48 \mathrm{~h}$, while the wounds in the APS groups remained unhealed at $48 \mathrm{~h}$ (fig. 2).

The number of migrating A549 cells was greatly reduced after treatment with APS ( $\mathrm{p}<0.05)$, which was reduced more significantly with the increase in the concentration of APS ( $<<0.05)$ (fig. 3). The number of invasive A549 cells was greatly reduced after treatment with APS $(p<0.05)$, which was reduced more significantly with the increase in the concentration of APS $(p<0.05)$ (fig. 4). After the A549 cells were treated with APS at different concentrations, the relative protein expressions of SHP2 and SOCS3 rose significantly with the increase in the concentration of APS $(p<0.05)$, while the relative protein expression of PIAS1 presented no significant change ( $p>0.05$ ) (fig. 5).

Lung cancer is one of the malignant tumors with

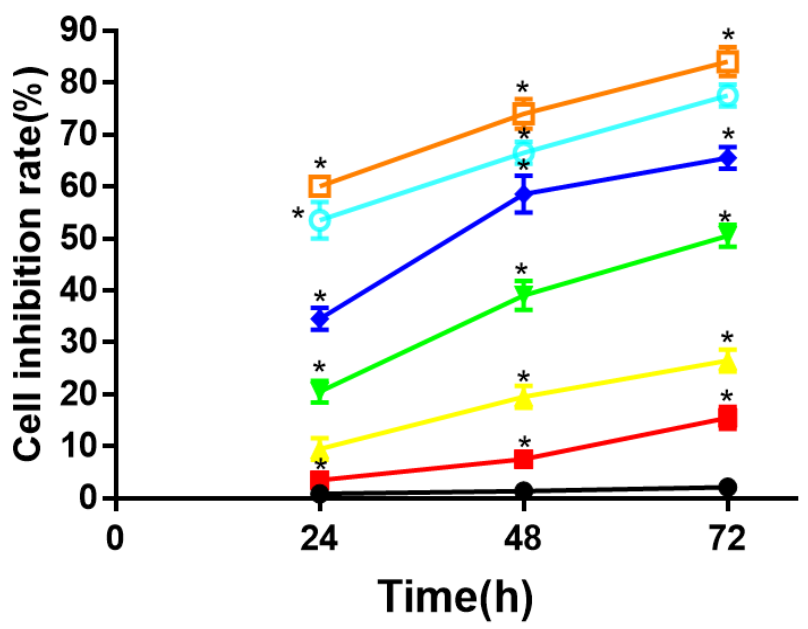

Fig. 1: Effects of APS on A549 cell proliferation, *p<0.05 vs. control group at the same time point; $(\neg-)$ : Control group; $(--): 0.5 \mathrm{mg} / \mathrm{ml} ;(--): 1.0 \mathrm{mg} / \mathrm{ml} ;(-\neg): 2.0 \mathrm{mg} / \mathrm{ml}$; $(\neg): 4.0 \mathrm{mg} / \mathrm{ml} ;(--)$ : $8.0 \mathrm{mg} / \mathrm{ml} ;(\square-)$ ): $16.0 \mathrm{mg} / \mathrm{ml}$ 


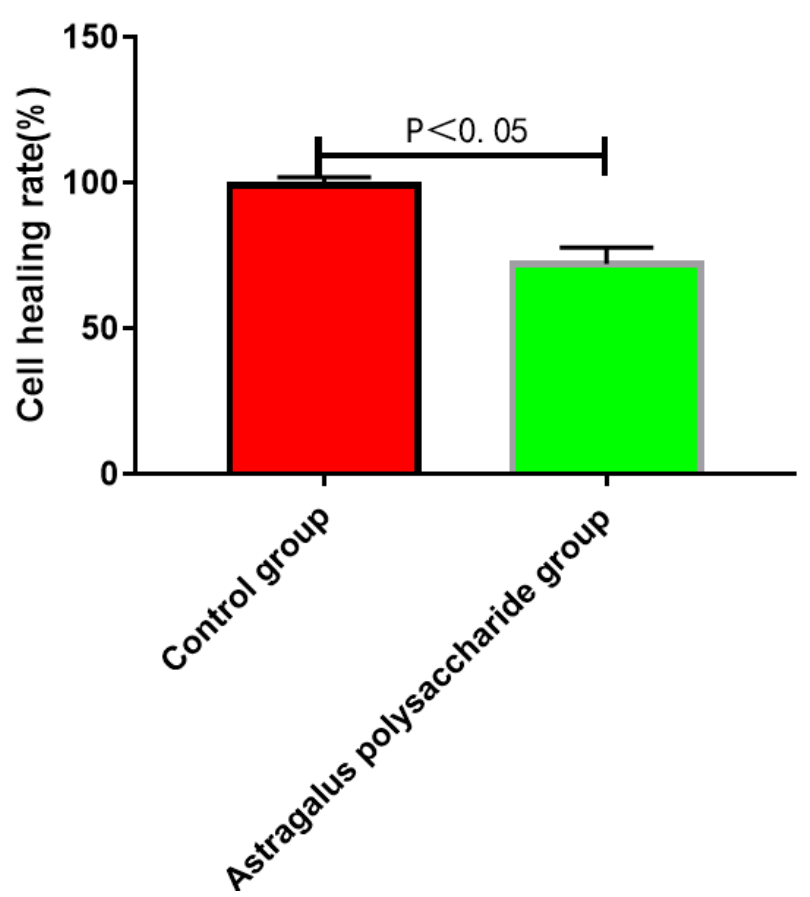

Fig. 2: Effects of APS on A549 cell wound healing

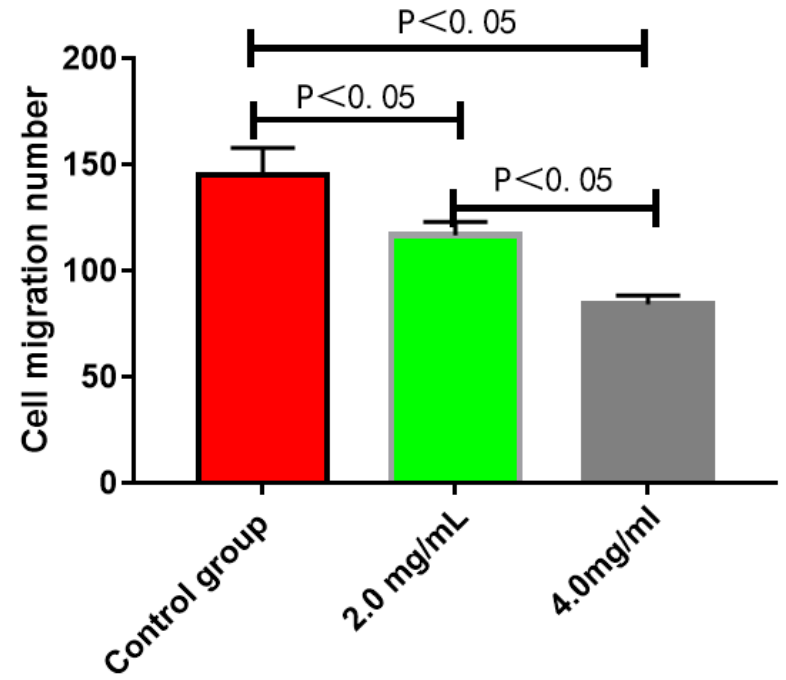

Fig. 3: Effects of APS on migration ability of A549 cells

high incidence and mortality in recent years, so it is particularly important to find out a chemotherapy drug that can efficiently kill and inhibit tumor cells while bringing no toxicity to normal cells or protecting normal cells ${ }^{[7]}$. With the continuous deepening of studies on modern pharmacology and the updating of extraction technologies of TCM, the anti-tumor mechanism of the effective components in traditional Chinese medicine has been clarified constantly. The TCM Astragalus membranaceus shows such broad pharmacological activities, and many studies have demonstrated that APS has significant anti-tumor effects $^{[8,9]}$. The Janus kinase/signal transducers and activators of transcription (JAK/STAT) cell signaling pathway is a pathway where various cytokines perform intracellular and extracellular signaling. Multiple studies ${ }^{[10]}$ have suggested that the JAK-STAT signaling pathway is widely involved in cell proliferation, differentiation and apoptosis, and the over-activation of the JAK-STAT cell signaling pathway is one of the main mechanisms causing neoplasia. However, there have been no relevant studies or reports on the effects of APS on the signaling pathway and its mechanism on lung cancer cells. For this reason, the inhibitory effects of APS on lung cancer cell proliferation were explored and analyzed in this study based on the regulatory mechanism of the JAK-STAT cell signaling pathway. The JAK-STAT cell signaling pathway is mainly regulated by three protein families and SHP2, SOCS3 and PIAS1, which are highly expressed in lung cancer cells, were analyzed in this study as the representative of each protein family.

The results of this study revealed that APS can inhibit the proliferation, migration and invasion of A549 lung cancer cells significantly and the effects are highly related to the concentration and action time of APS. After intervention with APS, the wound healing speed of A549 lung cancer cells was lower in APS groups than that in control group. The cells in control group almost fully healed after $48 \mathrm{~h}$ while the wounds in the APS groups remained unhealed at $48 \mathrm{~h}$. It has been further proved that APS can inhibit the migration of A549 lung cancer cells effectively, which is similar to the results of other related study reports ${ }^{[11,12]}$. APS shows significant anti-tumor activities due to its effective inhibition of proliferation, migration and invasion of lung cancer cells. Moreover, the effects of APS on the JAK-STAT cell signaling pathway of lung cancer cells were analyzed. SHP2 is one of the negative regulatory proteins of the JAK-STAT cell signaling pathway

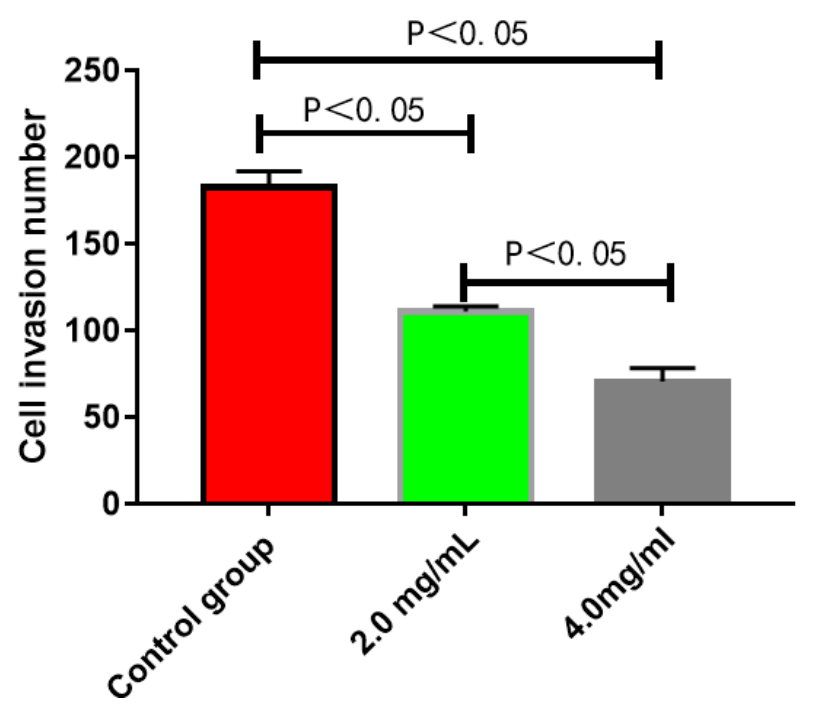

Fig. 4: Effects of APS on invasive ability of A549 cells 
A

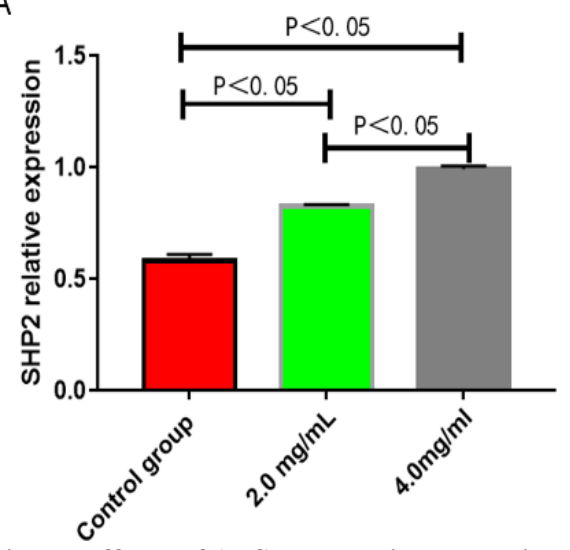

B

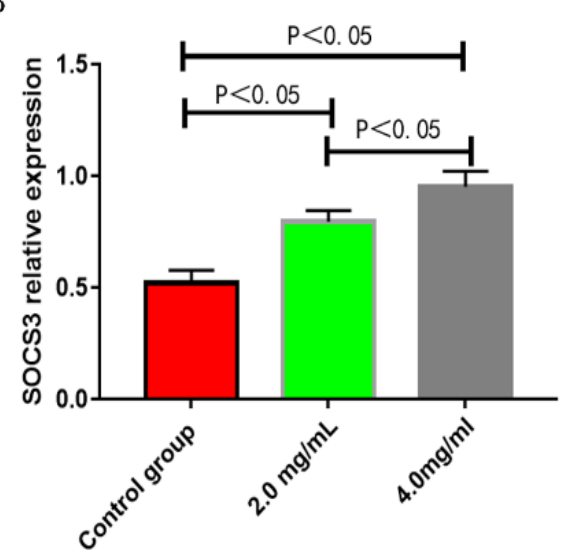

C

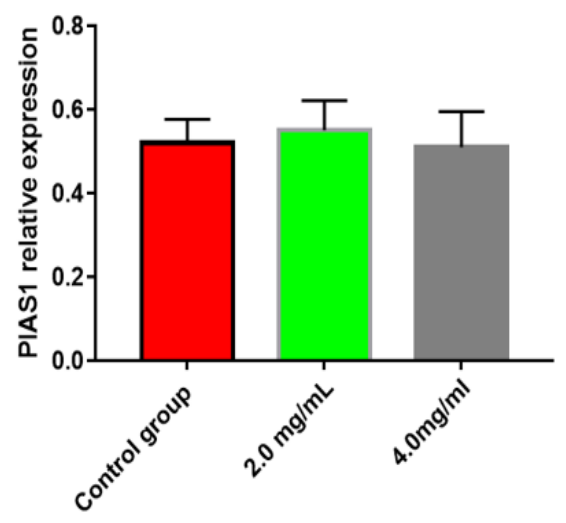

Fig. 5: Effects of APS on protein expressions of (A) SHP2; (B) SOCS3 and (C) PIAS1 in A549 cells

induced by interleukin 6(IL-6), but its specific molecular mechanism of action remains unclear. Scholars have verified that during the occurrence and development of liver cancer, SHP2 protein can inhibit the occurrence and development of liver cancer by dephosphorylation of signal transducer and activator of transcription 3 (STAT3) ${ }^{[13,14]}$. SOCS3, as one of the most important members of the suppressor of cytokine signaling (SOCS) protein family, as well as the most important inhibitory factor in the JAK-STAT cell signaling pathway, can be induced by multiple cytokines such as granulocyte colony-stimulating factor, erythropoietin and IL-6, and has a negative regulatory effect on the JAK-STAT cell signaling pathway ${ }^{[15]}$. According to the results of this study, after 549 cells were treated with APS at different concentrations, the relative protein expressions of SHP2 and SOCS3 rose significantly with the increase of the concentration of APS, indicating that the protein translation and expression of SHP2 and SOCS3 can be up-regulated after the intervention with APS on lung cancer cells, thus negatively regulating the JAK-STAT signaling pathway and inhibiting its over-activation, and thereby exerting the corresponding anti-tumor effects. PIAS is an inhibitor of activated STAT protein, which only inhibits the protein transcriptional activity of activated STAT, but has no inhibitory effect on other components of the JAK-STAT signaling pathway and the intracellular activated protein of the pathway ${ }^{[16-18]}$, which was further verified in this study. The results of this study showed that intervention with APS had no significant effect on the relative protein expression of PIAS1.

In conclusion, APS can significantly inhibit the proliferation of A549 lung cancer cells in a concentration- and time-dependent manner. In addition, APS can inhibit the migration and invasion of A549 lung cancer cells effectively and its mechanism of action may be related to the up-regulation of protein expressions of SHP2 and SOC3 in A549 lung cancer cells and the inhibition of over-activation of the JAKSTAT cell signaling pathway.

\section{Author contributions:}

Hanjiong Cai and Jianbiao Cao contributed equally to this study.

\section{Conflict of interests:}

The authors declared no conflicts of interest.

\section{REFERENCES}

1. Ferlay J, Shin HR, Bray F, Forman D, Mathers C, Parkin DM. Estimates of worldwide burden of cancer in 2008: GLOBOCAN 2008. Int J Cancer 2010;127(12):2893-917.

2. Parsons MW, Whipple NS, Poppe MM, Mendez JS, Cannon DM, Burt LM. The use and efficacy of chemotherapy and radiotherapy in children and adults with pilocytic astrocytoma. J Neurooncol 2021;151(2):93-101.

3. Daugèlaitė G, Užkuraitytė K, Jagelavičienė E, Filipauskas A. Prevention and treatment of chemotherapy and radiotherapy induced oral mucositis. Medicina 2019;55(2):25.

4. Leng J, Lei L, Lei SF, Zhu Z, Ocampo A, Gany F. Use of Traditional Chinese Herbal Medicine Concurrently with Conventional Cancer Treatment Among Chinese Cancer Patients. J Immigr Minor Health 2020;22(6):1240-7.

5. Sui X, Zhang M, Han X, Zhang R, Chen L, Liu Y, et al. Combination of traditional Chinese medicine and epidermal growth factor receptor tyrosine kinase inhibitors in the treatment of non-small cell lung cancer: A systematic review and meta-analysis. Medicine 2020;99(32):e20683.

6. Wu CY, Ke Y, Zeng YF, Zhang YW, Yu HJ. Anticancer activity of Astragalus polysaccharide in human non-small cell lung cancer cells. Cancer Cell Int 2017;17(1):115.

7. Kanda S, Goto K, Shiraishi H, Kubo E, Tanaka A, Utsumi $\mathrm{H}$, et al. Safety and efficacy of nivolumab and standard chemotherapy drug combination in patients with advanced non-small-cell lung cancer: a four arms phase Ib study. Ann Oncol 2016;27(12):2242-50.

8. Ye N, Cruz J, Peng X, Ma J, Zhang A, Cheng X. Remyelination is enhanced by Astragalus polysaccharides through inducing the differentiation of oligodendrocytes from neural stem 
cells in cuprizone model of demyelination. Brain Res 2021;1763:147459.

9. Ding $\mathrm{G}$, Gong Q, Ma J, Liu X, Wang Y, Cheng X. Immunosuppressive activity is attenuated by Astragalus polysaccharides through remodeling the gut microenvironment in melanoma mice. Cancer Sci 2021;112(10):4050-63.

10. van der Zee M, Sacchetti A, Cansoy M, Joosten R, Teeuwssen M, Heijmans-Antonissen C, et al. IL6/JAK1/STAT3 signaling blockade in endometrial cancer affects the ALDHhi/CD126+ stem-like component and reduces tumor burden. Cancer Res 2015;75(17):3608-22.

11. Zhao L, Zhong Y, Liang J, Gao H, Tang N. Effect of Astragalus polysaccharide on the expression of VEGF and EGFR in mice with Lewis transplantable lung cancer. J Coll Physicians Surg Pak 2019;29(4):392-4.

12. Tsao SM, Wu TC, Chen J, Chang F, Tsao T. Astragalus Polysaccharide Injection (PG2) Normalizes the Neutrophilto-Lymphocyte Ratio in Patients with Advanced Lung Cancer Receiving Immunotherapy. Integr Cancer Ther 2021;20.

13. Chang WH, Lai AG. An immunoevasive strategy through clinically-relevant pan-cancer genomic and transcriptomic alterations of JAK-STAT signaling components. Mol Med
2019;25(1):46.

14. Lv J, Yu W, Zhang Y, Cao X, Han L, Hu H, et al. LNK promotes the growth and metastasis of triple negative breast cancer via activating JAK/STAT3 and ERK1/2 pathway. Cancer Cell Int 2020;20:124.

15. Bagratuni T, Mavrianou N, Gavalas NG, Tzannis K, Arapinis $\mathrm{C}$, Liontos M, et al. JQ1 inhibits tumour growth in combination with cisplatin and suppresses JAK/STAT signalling pathway in ovarian cancer. Eur J Cancer 2020;126:125-35.

16. Dolatabadi S, Jonasson E, Lindén M, Fereydouni B, Bäcksten $\mathrm{K}$, Nilsson $\mathrm{M}$, et al. JAK-STAT signalling controls cancer stem cell properties including chemotherapy resistance in myxoid liposarcoma. Int J Cancer 2019;145(2):435-49.

17. Tabassum S, Abbasi R, Ahmad N, Farooqi AA. Targeting of JAK-STAT signaling in breast cancer: therapeutic strategies to overcome drug resistance. Adv Exp Med Biol 2019;1152:27181.

18. Liu ZK, Li C, Zhang RY, Wei D, Shang YK, Yong YL, et al. EYA2 suppresses the progression of hepatocellular carcinoma via SOCS3-mediated blockade of JAK/STAT signaling. Mol Cancer 2021;20(1):79. 